\title{
Tight control of light trapping in surface addressable photonic crystal membranes: application to spectrally and spatially selective optical devices
}

\author{
Cédric Blanchard ${ }^{1}$, Christian Grillet ${ }^{1}$, Cécile Jamois ${ }^{2}$, Jean-Louis Leclercq ${ }^{1}$, Pierre \\ Viktorovitch $^{1}$, and Xavier Letartre ${ }^{1}$ \\ ${ }^{1}$ Université de Lyon, Institut des Nanotechnologies de Lyon (INL), UMR CNRS 5270, Ecole Centrale de Lyon, \\ France \\ ${ }^{2}$ Université de Lyon, Institut des Nanotechnologies de Lyon (INL), UMR CNRS 5270, Institut National des \\ Sciences Appliqués de Lyon, France
}

Surface addressable Photonic Crystal Membranes (PCM) are 1D or 2D photonic crystals formed in a slab waveguides where Bloch modes located above the light line are exploited. These modes are responsible for resonances in the reflection spectrum whose bandwidth can be adjusted at will. These resonances result from the coupling between a guided mode of the membrane and a freespace mode through the pattern of the photonic crystal. If broadband, these structures represent an ideal mirror to form compact vertical microcavity with 3D confinement of photons [1] and polarization selectivity[2]. Among numerous devices, low threshold VCSELs with remarkable and tunable modal properties have been demonstrated. Narrow band PCMs (or high Q resonators) have also been extensively used for surface addressable optoelectronic devices where an active material is embedded into the membrane, leading to the demonstration of low threshold surface emitting lasers [3], nonlinear bistables [4], optical traps [5]...
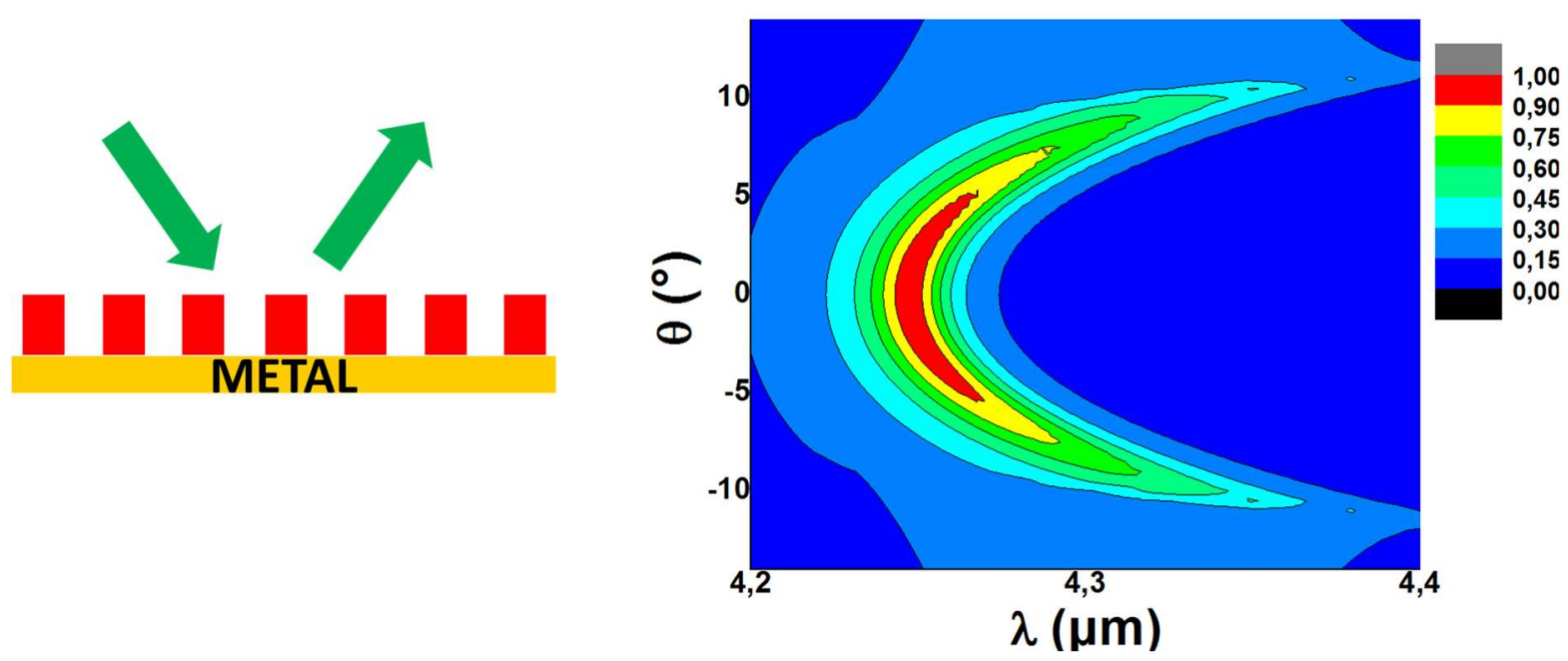

Figure 1: Spectral and angular dependence of the absorbance (emittance) of a 1D PCM deposited on a metal plate

In this presentation, we will describe the main physical rules which govern the lifetime of photons in these resonant modes. More specifically, it will be emphasized that the $Q$ factor of the PCM is determined, to the first order, by the integral overlap between the electromagnetic field distributions of the guided and free space modes and of the dielectric periodic perturbation which is applied to the homogeneous membrane to get the photonic crystal [6]. It turns out that the 
symmetries of these distributions are of prime importance for the strength of the resonance. It will be shown that, by molding in-plane or vertical symmetries of Bloch modes, spectrally and spatially selective light absorbers or emitters can be designed. First proof of concept devices will be also presented.

[1] P. Viktorovitch, B. Ben Bakir, S. Boutami, J.-L. Leclercq, X. Letartre, P. Rojo-Romeo, C. Seassal, M. Zussy, L. Di Cioccio, and J.-M. Fedeli, "3D harnessing of light with 2.5D photonic crystals," Laser \& Photon. Rev. 4, 401-413 (2010).

[2] S. Boutami, B. B. Bakir, H. Hattori, X. Letartre, J.-L. Leclercq, P. Rojo-Romeo, M. Garrigues, C. Seassal, and P. Viktorovitch, "Broadband and compact 2-D photonic crystal reflectors with controllable polarization dependence," IEEE Photon. Technol. Lett. 18, 835-838 (2006).

[3] J. Mouette, C. Seassal, X. Letartre, P. Rojo-Romeo, J.-L. Leclercq, P. Regreny, P. Viktorovitch, E. Jalaguier, P. Perreau, H. Moriceau, "Low threshold, room temperature vertical emitting laser based on an InP two-dimensional photonic crystal," Electron. Lett. 39, 526 (2003).

[4] C. Cojocaru, F. Raineri, P. Monnier, C. Seassal, X. Letartre, P. Viktorovitch, A. Levenson, R. Raj, "Ultrafast dynamics of the third-order nonlinear response in a two-dimensional InP-based photonic crystal," Appl. Phys. Lett. 85, 1880 (2004)

[5] L. Milord, E. Gerelli, C. Jamois, A. Harouri, C. Chevalier, P. Viktorovitch, X. Letartre, T. Benyattou, "Engineering of slow Bloch modes for optical trapping", Applied Physics Letters 106, 121110 (2015)

[6] C. Blanchard, P. Viktorovitch, X. Letartre, "Perturbation approach for the control of the quality factor in photonic crystal membranes: Application to selective absorbers", Physical Review A 90, 033824 (2014) 